\title{
V1016 CYG: PROPER MOTION OF RADIO EMISSION
}

\author{
S. P. S. EYRES ${ }^{1}$ S. M. DOUGHERTY ${ }^{2}$ R. J. DAVIS,${ }^{1}$ \\ M. F. BODE, ${ }^{2}$ H. M. LLOYD ${ }^{2}$ H. T. KENNY ${ }^{3}$ \\ 1. University of Manchester, NRAL, Jodrell Bank, \\ Macclesfield, Cheshire, SK11 9DL, UK \\ 2. Astrophysics Group, Liverpool John Moores University, \\ Liverpool, L3 $3 A F$, UK \\ 3. Royal Military College, Kingston, Canada
}

\begin{abstract}
We present MERLIN maps of V1016 Cyg at $6 \mathrm{~cm}$, observed on 1992 July 21 and 1995 March 23 . Previously discovered bipolar structure is resolved into three distinct peaks. Evolution and proper motion of these peaks has been measured.
\end{abstract}

\section{Discussion}

The symbiotic nova V1016 Cyg is one of the strongest radio emitters in that class of stars, HM Sge being the only comparable radio source of this type in the northern hemisphere (see Eyres et al. 1995a). Following optical outburst in 1965 (FitzGerald et al. 1966), V1016 Cyg has been well observed in the radio and Harris \& Scott (1976) first resolved a shell, believing it to have a bipolar appearance. Here we present MERLIN observations at $6 \mathrm{~cm}$ made on 1992 July 21 and 1995 March 23 . The bipolar features are resolved into three peaks, and the evolution of these peaks can be followed.

Fig. 1 shows the maps resulting from each observation. We identify three components in the earlier map which can be traced to the later one. The northern ridge is labelled $\mathrm{N}$, and contracts between the two observations. The southern features are labelled SE and SW. The SE feature appears to enlarge, and brightens overall, while the SW feature moves significantly between the two maps. Along with data at $18 \mathrm{~cm}, \mathrm{~V} 1016 \mathrm{Cyg}$ has many similarities in detailed radio structure to HM Sge (Eyres et al. 1995b).

Over the $975 \mathrm{~d}$ between observations, we have been able to measure proper motions of three identifiable features in the data. These are given in Table 1. Given the errors, only SW can be said to have moved with any

A. Evans and J. H. Wood (eds.), Cataclysmic Variables and Related Objects, 333-334. (C) 1996 Kluwer Academic Publishers. Printed in the Netherlands. 

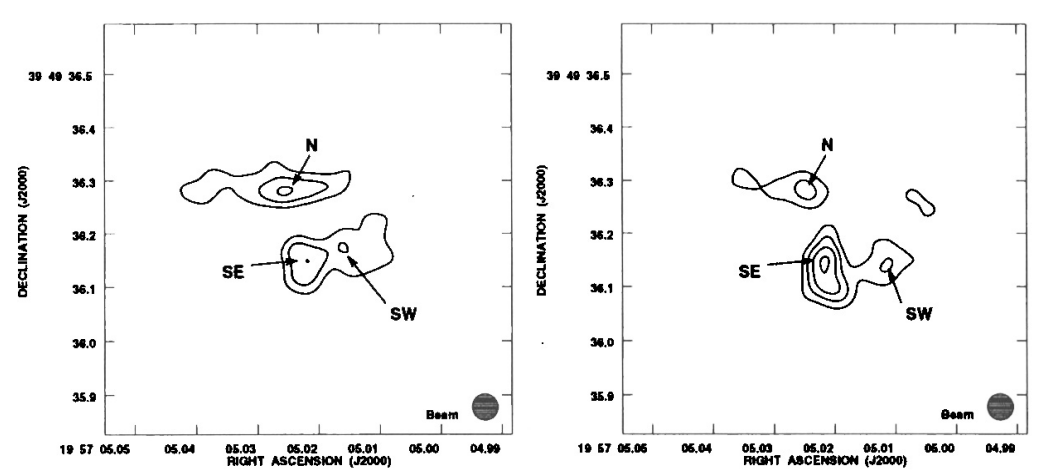

Figure 1. Radio maps of V1016 Cyg made using MERLIN at $6 \mathrm{~cm}$ on 1992 July 21 (left) and 1995 March 23 (right). Contour levels are 4, 6, 8 and 10 times the rms noise of $135 \mu \mathrm{Jy}$ beam $^{-1}$ The three features are labelled as $\mathrm{N}, \mathrm{SE}$, and $\mathrm{SW}$, for reference to Table 1.

TABLE 1. Proper motion in milli-arcsec (mas), position angle measured from north through east. Errors were $\sim 10$ mas in the positions from which the motions have been derived.

\begin{tabular}{lcccc}
\hline Feature & $\begin{array}{c}\Delta \mathrm{RA} \\
(\mathrm{mas})\end{array}$ & $\begin{array}{c}\Delta \text { dec } \\
(\mathrm{mas})\end{array}$ & $\begin{array}{c}\text { Total Motion } \\
(\mathrm{mas})\end{array}$ & Position Angle \\
\hline $\mathrm{N}$ & -15 & 0 & 15 & $270^{\circ}$ \\
$\mathrm{SE}$ & 0 & 0 & 0 & - \\
$\mathrm{SW}$ & -60 & -45 & 75 & $233^{\circ}$ \\
\hline
\end{tabular}

certainty. The association of the various radio components with the stellar components or outflow will require a more detailed analysis.

Acknowledgements. MERLIN is a national facility operated by the University of Manchester on behalf of PPARC. HML and SMD are supported by a Research Assistantships, and SPSE is supported by a Research Studentship Award from PPARC.

\section{References}

Eyres, S.P.S., Kenny, H.T., Cohen, R.J., et al., 1995a, MNRAS, 274, 317

Eyres, S.P.S., Davis, R.J., Kenny, H.T., et al., 1995b, in "Radio Emission from the Sun and Stars", eds. Taylor A.R., Paredes J.M., A. S. P., San Francisco, in press

FitzGerald, M.P., Houk, N., McCuckey, S.W., Hoffleit, D., 1966, Ap. J., 144, 1135

Harris, S., Scott, P.F., 1976, MNRAS, 175, 371 